\title{
27.
}

\section{NOUVELLES REMARQUES SUR LES COURBES DU TROISIÈME ORDRE.}

[From the Journal de Mathématiques Pures et Appliquées (Liouville), tom. x. (1845), pp. 102-109.].

JE me propose de développer ici quelques conséquences de la théorie que j’ai donnée, il y a quelques mois, dans ce Journal ${ }^{1},[26]$, des points correspondants des courbes du troisième ordre. Rappelons d'abord la signification de ce terme et ajoutons-y quelques nouvelles définitions.

On dit que les points $A, A^{\prime}$ sont correspondants quand les tangentes à la courbe en ces points se rencontrent sur la courbe. En considérant les points correspondants $A, A^{\prime}$ et les deux autres points correspondants $B, B^{\prime}$, on dit que ces paires correspondent, quand les points d'intersection $H, h$ de $A B^{\prime}, A^{\prime} B$ ou $A B, A^{\prime} B^{\prime}$ sont situés sur la courbe. Les trois paires $A, A^{\prime}, B, B^{\prime}, H, h$ sont nommées paires supplémentaires ou système supplémentaire. On dirait de même que les deux systèmes $A A^{\prime}, B B^{\prime}, H h$ et $A_{1} A_{1}^{\prime}, B_{1} B_{1}^{\prime}, H_{1} h_{1}$ sont des systemes supplémentaires correspondants, si, par exemple, $A, A^{\prime}$ et $A_{1}, A_{1}^{\prime}$, \&c., formaient des paires correspondantes.

On peut nommer quadrilatère inscrit le système de quatre droites qui passent par les points supplémentaires $A A^{\prime}, B B^{\prime}, H h$, et conique d'involution chaque conique tangente à ces quatre droites, ou, en d'autres termes, inscrite dans un quadrilatère inscrit. Il est inutile d'expliquer ce que veulent dire coniques d'involution correspondantes, ou quadrilatères correspondants, ou l'expression conique correspondante à une paire donnée de points correspondants, \&c.

Démontrons les théorèmes suivants :

THÉORÈme I. "Les tangentes menées par un point $P$ de la courbe à trcis coniques d'involution correspondantes forment un faisceau en involution."

1 Voyez page 285 du tome Ix. 
Chaque conique peut se réduire à une paire de points qui correspondent aussi aux coniques (ce qui justifie la dénomination que nous avons donnée à ces coniques). Considérons un quadrilatère inscrit $A A^{\prime}, B B^{\prime}, H h$, la conique d'involution tangente aux côtés de ce quadrilatère, et deux paires $L L^{\prime}, M M^{\prime}$ de points correspondants, ces paires étant correspondantes l'une à l'autre et à la conique d'involution. On sait que les lignes menées d'un point quelconque, et ainsi du point $P$ de la courbe, par les points $A, A^{\prime}, B, B^{\prime}$, forment avec les tangentes à la conique menées par ce même point, un faisceau en involution. Mais $P A, P A^{\prime}, P B, P B^{\prime}, P L, P L^{\prime}$, et de même $P B, P B^{\prime}$, $P L, P L^{\prime}, P M, P M^{\prime}$, forment aussi des faisceaux en involution; donc les deux tangentes forment, avec $P L, P L^{\prime}$ et $P M, P M^{\prime}$, un faisceau en involution. De même, avec une conique correspondante à la première, $P L, P L^{\prime}, P M, P M^{\prime}$ et les deux tangentes forment un faisceau en involution; donc $P L, P L^{\prime}$ et les quatre tangentes forment un faisceau en involution, et de même en introduisant la troisième conique.

THÉORÈME II. "On peut circonscrire à une conique d'involution donnée une infinité de quadrilatères inscrits correspondants au premier."

Soient $A, A^{\prime}, B, B^{\prime}, H, h$ comme auparavant; et $A_{1}, A_{1}^{\prime}$ une paire de points correspondants qui correspondent à ceux-ci; en menant par $A_{1}, A^{\prime}{ }_{1}$ des tangentes à la conique qui se rencontrent en $B_{1}, B_{1}^{\prime}, H_{1}, h_{1}$, on voit d'abord que les lignes $P A, P A^{\prime}$, $P A_{1}, P A_{1}^{\prime}$ et les tangentes à la conique forment un faisceau en involution; et reciproquement, chaque point $P$ qui satisfait à cette condition appartient à la courbe. Mais en prenant, par exemple, pour $P$ le point $B_{1}$, les lignes $P A_{1}, P A^{\prime}{ }_{1}$ deviennent identiques avec les deux tangentes, ce qui satisfait à la condítion d'involution. Donc $B_{1}, B_{1}^{\prime}, H_{1}, h_{1}$ appartiennent à la courbe, ou $A_{1}, A_{1}^{\prime}, B_{1}, B_{1}^{\prime}, H_{1}, h_{1}$ forment un quadrilatère inscrit correspondant au premier ou à la conique.

ThÉorème III. "Les centres d'homologie de deux coniques d'involution correspondantes forment un quadrilatère inscrit correspondant aux coniques."

Considérons les deux coniques et une troisième conique quelconque. Chaque point $P$ pour lequel les six tangentes forment un faisceau en involution appartient à la courbe. En prenant pour $P$ un centre d'homologie des deux premières coniques, les deux paires de tangentes deviennent identiques, ce qui satisfait à la condition d'involution; donc les six centres d'homologie sont sur la courbe, ou ces six points sont les sommets d'un quadrilatère inscrit qui correspond aussi aux coniques.

\section{Réciproquement,}

THÉonغ̀me IV. "Le lieu d'un point $P$ qui se meut de manière que les tangentes menées par ce point à trois coniques données quelconques, forment toujours un faisceau en involution, est une courbe du troisième ordre qui passe par les dix-huit centres d'homologie des coniques prises deux à deux, ces centres formant six à six des quadrilatères inscrits correspondants."

La démonstration analytique de la première partie de ce théorème, quoique longue, me paraît assez intéressante pour trouver place ici. Pour plus de symétrie, prenons $\frac{\xi}{\zeta}, \frac{\eta}{\zeta}$ pour les coordonnées indéfinies d'un point, et représentons par

$$
\Upsilon=0, \quad \Upsilon^{\prime}=0, \quad \Upsilon^{\prime \prime}=0,
$$


les équations des trois coniques, $\Upsilon$, \&c. étant des fonctions homogènes de la forme

$$
\Upsilon=A \xi^{2}+B \eta^{2}+C \zeta^{2}+2 F \eta \zeta+2 G \zeta \xi+2 H \xi \eta, \& c .
$$

Soient $\frac{x}{z}, \frac{y}{z}$ les coordonnées du point $P$; mettons, pour abréger,

$$
\begin{aligned}
U & =A x^{2}+B y^{2}+C z^{2}+2 F y z+2 G z x+2 H x y, \\
W & =A x \xi+B y \eta+C z \zeta+F^{\prime}(y \zeta+\eta z)+G(z \xi+\zeta x)+H(x \eta+y \xi)
\end{aligned}
$$

(de manière que $W=0$ serait l'équation de la ligne polaire du point $P$ ). Nous avons

$$
U \Upsilon-W^{2}=0,
$$

pour l'équation des deux tangentes menées par le point $P$ à la conique. Cela est probablement connu. Il est clair d'abord que cette équation appartient à une conique qui a un double contact avec la conique donnée, et par la forme à laquelle nous allons réduire cette équation, on voit ensuite qu'elle appartient à un système de deux droites qui passent par le point $P$. En développant et écrivant

$$
\begin{array}{lll}
B C-F^{2}=a, & C A-G^{2}=b, & A B-H^{2}=c, \\
G H-A F=f, & H F-B G=g, & F G-C H=h,
\end{array}
$$

on trouve, en effet,

$$
\begin{aligned}
a(y \zeta-z \eta)^{2} & +b(z \xi-x \zeta)^{2}+c(x \eta-y \xi)^{2} \\
& +2 f(z \xi-x \zeta)(x \eta-y \xi)+2 g(x \eta-y \xi)(y \zeta-z \eta)+2 h(y \zeta-z \eta)(z \xi-x \zeta)=0,
\end{aligned}
$$

et de là, en posant

on obtient

$$
\begin{array}{ll}
\mathfrak{A}=b z^{2}+c y^{2}-2 f y z, & \mathbb{f} \mathfrak{f}=a y z-g x y-h x z+f x^{2}, \\
\mathfrak{A}=c x^{2}+a z^{2}-2 g x z, & \mathbf{G}=b z x-h y z-f x y+g y^{2}, \\
\mathfrak{C}=a y^{2}+b x^{2}-2 h x y, & \mathfrak{F}=c x y-f x z-g y z+h z^{2},
\end{array}
$$

$$
\mathfrak{A} \xi^{2}+2 \mathfrak{B} \eta^{2}+\mathfrak{C} \zeta^{2}-2 \sqrt{f} \eta \zeta-2 \mathscr{G} \xi \zeta-2 \mathfrak{z} \xi \xi \eta=0,
$$

ou, en transportant l'origine au point $P$,

$$
\mathfrak{A} \xi^{2}+\mathfrak{d} \boldsymbol{B} \eta^{2}-2 \mathfrak{z} \boldsymbol{Z} \xi \eta=0 ;
$$

et de même, pour l'équation des tangentes, par ce même point aux deux autres coniques

$$
\begin{aligned}
& \left.\mathfrak{A}^{\prime} \xi^{2}+\mathfrak{x}^{\prime} \eta^{2}-2\right] \\
& \mathfrak{A}^{\prime \prime} \xi^{2}+\mathbf{B b}^{\prime \prime} \eta^{2}-2 \mathbb{T}^{\prime \prime} \xi^{\prime \prime} \eta=0 \text {. }
\end{aligned}
$$

On obtient donc, pour la condition que ces lignes forment un faisceau en involution,

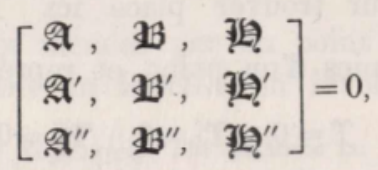


en représentant de cette manière le déterminant formé avec ces neuf quantités. Formons d'abord la fonction

$$
\mathfrak{A} \mathfrak{A} \mathfrak{B}^{\prime \prime}-\mathfrak{A} \mathfrak{A} \mathfrak{B}^{\prime \prime}
$$

Cela se reduit à

$$
z\left[-2(f c) x^{2} y-2(c g) x y^{2}+(c a) y^{2} z-2(f a) y z^{2}-2(b g) x z^{2}+(b c) x^{2} z+4(f g) x y z+(b a) z^{3}\right],
$$

où $(f c)=\left(f^{\prime} c^{\prime \prime}-f^{\prime \prime} c^{\prime}\right), \& c$.

Multipliant par 鸨, formant ensuite les quantités analogues et ajoutant; écrivant aussi

$$
c(f g)+c^{\prime}\left(f^{\prime} g^{\prime}\right)+c^{\prime \prime}\left(f^{\prime \prime} g^{\prime \prime}\right)=(c f g),
$$

c'est-à-dire $(c f g)$ pour le déterminant formé avec les neuf quantités

$$
c, f, g, \quad c^{\prime}, f^{\prime}, g^{\prime}, \quad c^{\prime \prime}, f^{\prime \prime}, g^{\prime \prime},
$$

on obtient d'abord les termes

$$
-4(c f g) x^{2} y^{2} z^{2}, \quad+2(f c g) x^{2} y^{2} z^{2}, \quad+2(g f c) x^{2} y^{2} z^{2},
$$

qui se détruisent; les autres termes contiennent $z^{3}$ comme facteur, et en écartant cette quantité, l'on obtient en dernière analyse l'équation

$$
\begin{aligned}
(c b f) x^{3} & +(a c g) y^{3}+(b a h) z^{3}+4(f g h) x y z \\
& +[2(a g f)+(c a h)] y^{2} z+[2(b h g)+(a b f)] z^{2} x+[2(c f h)+(b c g)] x^{2} y \\
& +[2(a f h)+(a b g)] y z^{2}+[2(b g f)+(b c h)] z x^{2}+[2(c h g)+(c a f)] x y^{2}=0
\end{aligned}
$$

(où l'on peut, si l'on veut, écrire $z=1$ ). C'est l'équation d'une courbe du troisième ordre.

ObServation. Cette méthode peut être utile dans l'investigation d'autres problèmes relatifs aux coniques. Par exemple, la question de déterminer les centres d'homologie de deux coniques données revient à celle-ci: satisfaire identiquement à l'équation

$$
\begin{aligned}
& \mathfrak{A} \xi^{2}+\mathfrak{B} \eta^{2}+\mathbb{C} \zeta^{2}+2 \sqrt{f} \eta \zeta+2 \mathbb{G} \xi \xi+27 \boldsymbol{Z} \xi \eta
\end{aligned}
$$

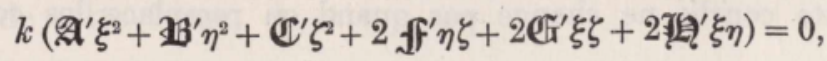

parce que, en effectuant cela, il est facile de voir que $\frac{x}{z}, \frac{y}{z}$ sont les coordonnées du centre cherché. Écrivons

$$
a+k a^{\prime}=\mathrm{a}, \quad c+k b^{\prime}=\mathrm{b}, \quad \& \mathrm{c} .
$$

l'on obtient les six équations

c.

$$
\begin{array}{ll}
\mathrm{b} z^{2}+\mathrm{c} y^{2}-2 \mathrm{f} y z=0, & \mathrm{a} y z-\mathrm{g} x y-\mathrm{h} x z+\mathrm{f} x^{2}=0, \\
\mathrm{c} x^{2}+\mathrm{a} z^{2}-2 \mathrm{~g} z x=0, & \mathrm{~b} z x-\mathrm{h} y z-\mathrm{f} y x+\mathrm{g} y^{2}=0, \\
\mathrm{a} y^{2}+\mathrm{b} x^{2}-2 \mathrm{~h} x y=0, & \mathrm{c} x y-\mathrm{f} z x-\mathrm{g} z y+\mathrm{h} \boldsymbol{c}^{2}=0,
\end{array}
$$


dont les trois dernières se déduisent des autres. On peut, de ces six équations, éliminer les six quantités $x^{2}, y^{2}, z^{2}, y z, z x, x y$, considérées comme indépendantes; on obtient ainsi, toute réduction faite,

$$
\left(a b c-a f^{2}-b g^{2}-c h^{2}+2 f g h\right)^{2}=0,
$$

équation qui détermine la quantité $k$; les trois premières équations deviennent alors équivalentes à deux, qui suffisent pour déterminer les rapports $\frac{x}{z}, \frac{y}{z}$. Il serait facile de rapprocher cette solution de celle que l'on déduit de la théorie des polaires reciproques. Par exemple, l'équation qui vient d'être obtenue entre les quantités $a, b, \ldots$ est précisément celle qui exprime que la fonction

$$
\mathrm{a} x^{2}+\mathrm{b} y^{2}+\mathrm{c} z^{2}+2 \mathrm{f} y z+2 \mathrm{~g} x z+2 \mathrm{~h} x y
$$

se divise en facteurs linéaires. Remarquons encore que, dans la géométrie solide, l'équation

$$
U \Upsilon-W^{2}=0
$$

appartient au cône, ayant le point $P$ pour sommet, et circonscrit à une surface du second ordre qui a pour équation $\Upsilon=0$. C'est un cas particulier d'une autre formule que voici :

“En représentant par 非 une fonction linéaire des trois variables $\xi, \eta, \zeta$ (ou de quatre variables sans termes constants), et par $P$ la même fonction de $x, y, z$, l'équation

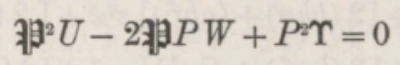

appartient au cône ayant pour sommet le point dont les coordonnées sont $x, y, z$, et passant par la courbe d'intersection du plan $P=0$, et de la surface du second ordre $\Upsilon=0$. Et de même pour deux variables."

Je finirai en citant un théorème de géométrie dû à $\mathbf{M}$. Hesse ${ }^{1}$, qui a quelques rapports avec le sujet que je viens de traiter:-

"Le lieu d'un point $P$, qui se meut de manière que ses polaires par rapport à trois coniques données se rencontrent dans le même point, est une courbe du troisième ordre; et encore cette courbe ne change pas quand on remplace les coniques données

$$
U=0, \quad U^{\prime}=0, \quad U^{\prime \prime}=0,
$$

par trois nouvelles coniques de la forme

$$
\lambda U+\lambda^{\prime} U^{\prime}+\lambda^{\prime \prime} U^{\prime \prime}=0 . "
$$

Cette courbe du troisième ordre est tout à fait distincte de celle que j'ai considérée.

1 Voyez le Journal de M. Crelle, tome xxvrr. 